\title{
Tourism impact on municipal solid waste: elaborations for the case study "Adriatic Riviera" (Province of Rimini, Italy)
}

\author{
C. Caramiello ${ }^{1}$, L. Fabbri ${ }^{2}$, M. Marzi ${ }^{1}$ \& F. Tatàno ${ }^{1}$ \\ ${ }^{I}$ Department "DiGeoTeCA", Science and Technology Faculty, \\ University of Urbino "Carlo Bo", Italy \\ ${ }^{2}$ HERA Rimini S.r.l., HERA Group, Rimini, Italy
}

\begin{abstract}
In sizeable tourist areas, the mutual interaction "tourism - municipal solid waste (MSW) - sustainability" should be attentively investigated, as tourism fluxes could have a strong impact on the performance of implemented MSW management strategies. In this perspective, the present paper analyses the possible tourism influence on MSW generation, composition and separate collection with reference to the Italian case study of "Adriatic Riviera" (expressly located in the Province of Rimini, Central Italy), which is internationally renowned as a major summer seaside destination. In order to evaluate effectively the tourism impact on MSW, the provincial territory of Rimini has been suitably divided into some "territorial macro-areas". The elaborations relate to: the monthly variations of MSW production, the estimate of resulting MSW per-capita production, the estimate of MSW compositions at generation source, the monthly variations of separate collection, and finally the estimate of resulting per-capita separate collection yields.
\end{abstract}

Keywords: impact, municipal solid waste, sustainable development, tourism.

\section{Introduction: sustainable MSW management and tourism}

At the European level, the concept of the "integrated" management of municipal solid waste (MSW) was effectively introduced with the specific Commission Communication of September 1989 on a Community strategy for waste management, which prescribed a hierarchic policy with waste prevention as the 
first priority, followed by recovery and finally by safe waste disposal [1]. Afterwards, the "integrated" hierarchic strategy for MSW management was confirmed by the European Commission Communication "Com (96) 399 final" [2], establishing however that - within the recovery principle - preference should in general be given to material recovery over energy recovery [1]. Lastly, the new European Waste Directive 2008/98/EC [3] has reorganised the "integrated" waste policy in the following hierarchic order: prevention; preparing for re-use; recycling; other recovery, e.g. energy recovery; and disposal.

Progressively, the subsequent correlation at the European level of the "sustainable development" concept with MSW, thus prefiguring an innovative strategy for "sustainable and integrated" MSW management [1], had a concrete starting-point at the European Council meeting in Göteborg (June 2001) properly stating that "the relation between economic growth, consumption of natural resources and the generation of waste must change. Strong economic performance must go hand in hand with sustainable use of natural resources and levels of waste" [4]. Similarly, at an international level the "WSSD, World Summit on Sustainable Development" (Johannesburg, 2002) specifically calls in Article 22 of its "Plan of Implementation" [5] - for the implementation of the hierarchic, integrated management strategy for MSW (prevention, reuse and recovery, environmentally sound disposal) as an opportunity for "changing unsustainable patterns of consumption and production". Simultaneously, this "Plan of Implementation" also calls (in its Article 43) for the promotion of sustainable tourism as a possible strategy for "protecting and managing the natural resource base of economic and social development". Consequently, the mutual interaction "tourism - MSW - sustainability" should be investigated in significant tourist areas, as the tourist fluxes could have a strong impact on the resulting performance of implemented MSW management strategies.

Within the above-synthesised perspective, this paper presents and analyses some elaborations on the possible touristic influence on the generation, composition and separate collection of MSW with reference to the coastal area of the "Adriatic Riviera" (located in the Province of Rimini, Central Italy), which is internationally renowned as a major summer seaside destination. The selected years of monitoring were: expressly 2006, and additionally 2004 and 2005 for some specific annual elaborations. This study can be properly compared and integrated with a previous Italian investigation concerning the summer touristic influence on MSW production, composition and separate collection in a different area characterized by a typical mountain tourism [6]: the "Sun Valley" district located in the Province of Trento (North-eastern Italy).

\section{Methodology of data collection and elaboration}

\subsection{Territorial subdivision and data collection}

For the elaborations of this study, the provincial territory of Rimini has been suitably divided into the following "territorial macro-areas" (Figure 1): 1) the "Coastal Municipalities" (no. 5 towns), directly located on the Adriatic seaside 
front and predominantly interested by the summer tourist flux (see Section 3.1); 2) the boundary belt of "Intermediate Municipalities" (no. 8 towns); 3) the internal, territorial portion of "Hilly Municipalities" (no. 7 towns); 4) for a general view, the overall "Provincial Territory". Regarding the adopted scheme (by the responsible multi-utility company: Hera Rimini S.r.1.) for MSW separate collection, the territorial collection system is based on the diffuse presence of "localised sets" of road containers, with differentiations on the types of collected recyclable fractions among the mentioned macro-areas. In addition, the provincial inhabitants have also the opportunity to delivery recyclable materials at an integrated network of definable "supervised eco-stations" (no. 13 in total) within the overall provincial territory. The monitoring data, officially collected for carrying out the elaborations of this study, consisted of: 1) the weight quantities of MSW fractions collected separately and of residual MSW, available on a monthly temporal basis for individual municipalities during the overall period 2004-2006; 2) with reference to the monitoring year 2006, the resident populations (for individual municipalities) and the recorded tourist fluxes as overnight stays (properly disaggregated for each town of the "Coastal Municipalities" and for the remaining "Hinterland" provincial territory, according to the following temporal sequence: Jan-Apr, May, Jun, Jul, Aug, Sept, Oct-Dec); 3) the experimental investigations on waste physical composition (as component percent by weight) carried out during representative days of the year 2005 respectively for a) the residual MSW feeding the provincial waste-to-energy plant (no. 2 investigations, in February and August) and $b$ ) the residual MSW from road containers located in the seaside touristic zone of two towns in the macro-area "Coastal Municipalities" (no. 2 investigations for each town in summer period, August and September).

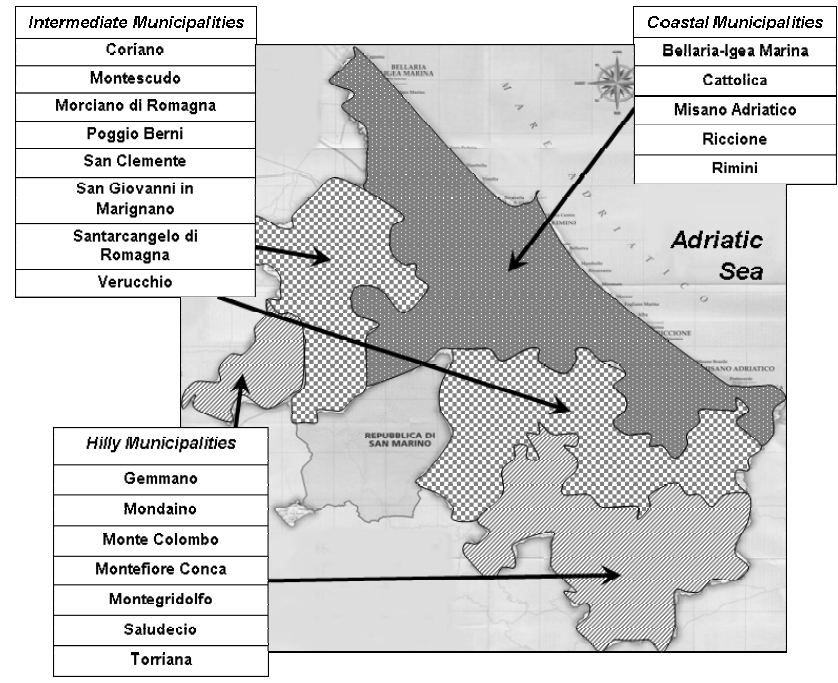

Figure 1: Territorial "macro-areas" of the Province of Rimini comparatively assumed in this study. 


\subsection{Elaboration procedures}

The data elaborations have been carried out in accordance with a proper sequential order. Firstly, due to the predominant tourism flux concentration in one of the territorial macro-areas (i.e., "Coastal Municipalities": see Section 3.1), the monthly variations of MSW production (during the monitoring year 2006) have been aggregated for each territorial macro-area.

With regard instead to the determination of the MSW per-capita production (as properly evaluated on a temporal annual basis [12]) referable on the whole to a given territorial area, two alternative approaches have been considered for the assumption on the representative population: 1) for each territorial macro-area (see Section 2.1), the consideration solely of the "resident" (inhabitant) population, thus independent of the touristic fluxes; 2) for the areas "Coastal Municipalities", "Hinterland" (see Section 2.1) and "Province of Rimini", the alternative consideration of the definable "total yearly equivalent" population, as derived from the sum of the "resident" population and the definable "yearly equivalent touristic" population. The units of "yearly equivalent tourist" have been achieved from the ratio between the yearly touristic overnight stays and the days in one year $[7,8]$. Thus, the "yearly equivalent tourist" is considered on an annual average [8] and is not properly assimilable to the inhabitant, because he has a peculiar impact on the territory especially in terms of resource consumption and consequential waste generation. Moreover, the sequential calculation approach of Figure 2 has permitted to disaggregate (for the monitoring year 2006) the MSW per-capita productions individually attributable to the typical "resident" person and the "yearly equivalent tourist" person in the macro-area "Coastal Municipalities", which is strongly influenced by the intense summer tourism. The starting point of this approach has been the assumption, as representative MSW per-capita production for the mentioned macro-area, of the average MSW per-capita production resulting for those municipalities of the macro-area "Intermediate Municipalities (with a negligible tourism impact: see Section 3.1) properly located on the boundary of the coastal towns.

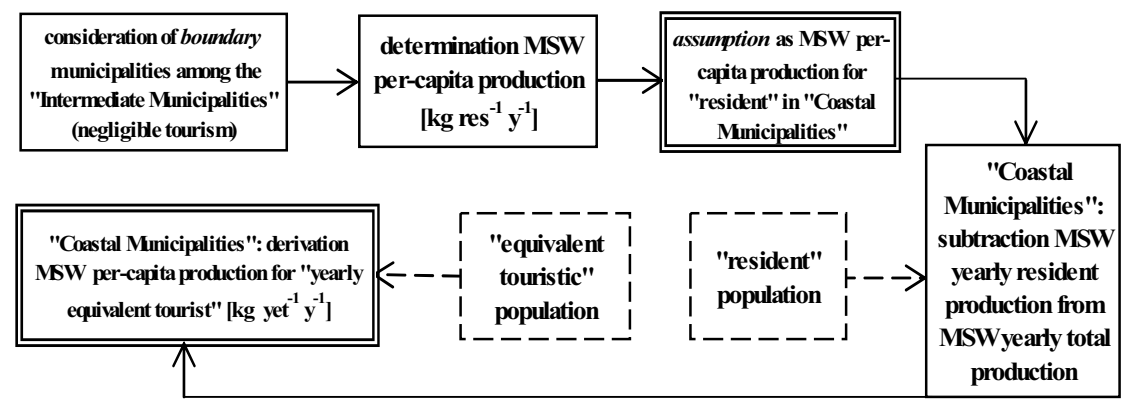

Figure 2: $\quad$ Macro-area "Coastal Municipalities", 2006: applied procedure for the determination of MSW per-capita productions attributable to the "resident" person and the "yearly equivalent tourist" person. Legend: $y=$ year; res = resident; yet $=$ yearly equivalent tourist. 


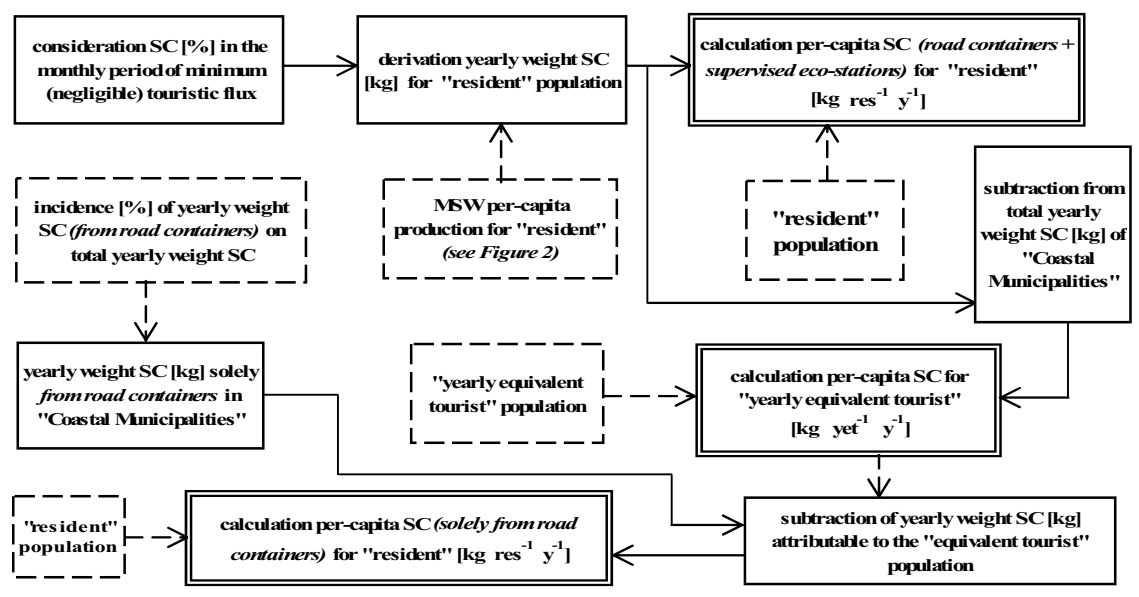

Figure 3: Macro-area "Coastal Municipalities", 2006: applied procedure for the determination of per-capita waste separate collection (SC) yields attributable to the "resident" person and the "yearly equivalent tourist" person. Legend: $y$, res, yet $=$ see Figure 2 .

In order to estimate the possible touristic summer influence on MSW composition at the generation source, the weight quantities of residual MSW components derivable from the available experimental investigations on physical composition (see Section 2.1) have been properly aggregated with the corresponding weight quantities of MSW components collected separately in the specific macro-area "Coastal Municipalities". This aggregation procedure has conclusively permitted the calculation - for this touristic macro-area "Coastal Municipalities" - of MSW physical compositions (as percent by weight) resulting at the generation source representatively for a winter month (Feb 2005), the summer two-months (Aug-Sep 2005) and the overall year (2005).

With final regard to the possible tourism impact on the separate collection of MSW, the following elaborations have been developed: 1) the aggregation of the resulting monthly separate collection rates (as both weight quantity and efficiency percentage, during 2006) for each territorial macro-area; 2) according to the calculation approach of Figure 3, the estimate (for the year 2006) of the per-capita separate collection yields (as weight quantity) individually attributable to the "resident" person (either considering the overall waste collection scheme or solely the road container collection scheme: see Section 2.1) and the "yearly equivalent tourist" person in the touristic macro-area "Coastal Municipalities".

\section{Results and discussion}

\subsection{Resident population and tourism distributions}

Referring to the monitoring year 2006, the "resident" population in the Province of Rimini (in total, 294,110 inhabitants) is predominantly concentrated in the 
macro-area "Coastal Municipalities" (with a resulting rate of $73.9 \%$ ), probably because of the consistent working offer which represents a strong attraction for migratory flows. The remaining inhabitant distribution rates are $21.9 \%$ for the macro-area "Intermediate Municipalities" and 4.2\% for the macro-area "Hilly Municipalities".

During the same monitoring year 2006, the macro-area "Coastal Municipalities" has collected the predominant $99.4 \%$ rate of the total provincial touristic flux (as 15,455,703 visitors in terms of overnight stays, corresponding to 42,344 individuals of total "yearly equivalent tourist" provincial population). The negligible $0.6 \%$ rate of the total tourist flux is referred instead to the remaining "Hinterland" territory (see Section 2.1). Regarding the temporal distribution of the tourist flux in the macro-area "Coastal Municipalities", the highest values of visitors, as shown in the aggregate diagram of Figure 4, are considerably concentrated during the summer season (June - September).

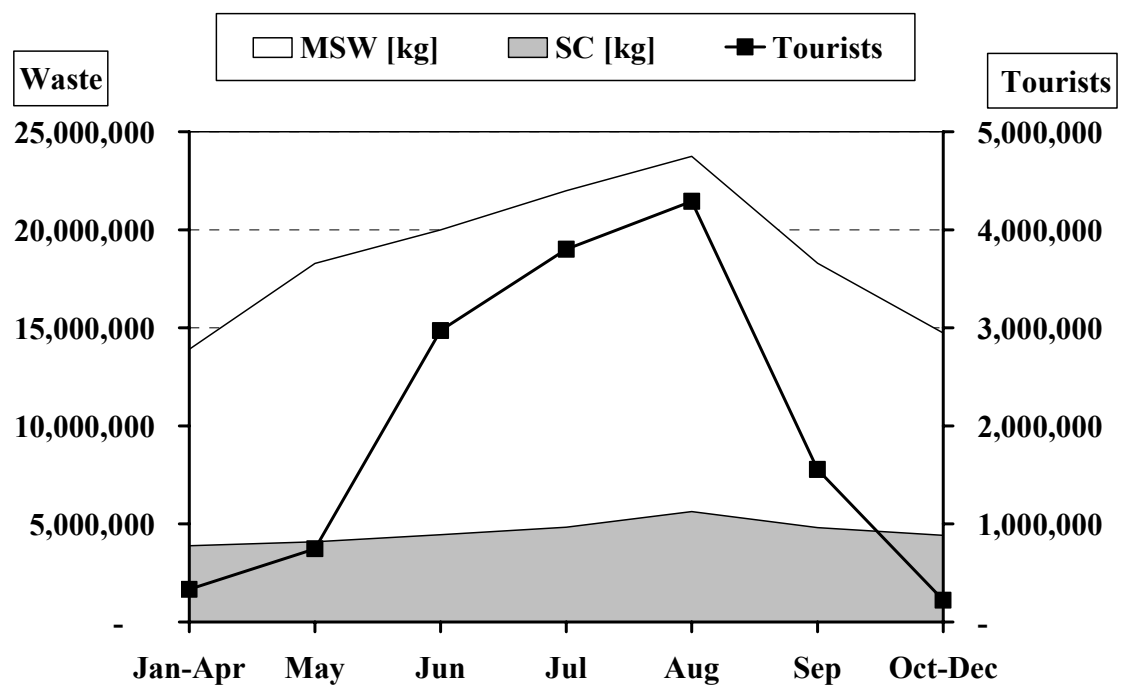

Figure 4: Qualitative comparison of the monthly evolutions of MSW production, tourist flux (as overnight stays) and separate collection quantity (SC) in the macro-area "Coastal Municipalities", monitoring year 2006. Due to the temporal aggregation form available for tourist fluxes (see Section 2.1), the data for Jan-Apr and Oct-Dec represent the respective monthly means.

\subsection{MSW production, composition and separate collection}

The monthly evolutions of MSW production, resulting for the monitoring year 2006, are available in the aggregate diagrams of Figure 5. The mutual comparison of these temporal evolutions among the macro-areas "Coastal Municipalities", "Intermediate Municipalities" and "Hilly Municipalities", 
shows solely in the specific area "Coastal Municipalities" a strong and clear fluctuation between the winter condition of min MSW production and the summer time of max MSW production, with a resulting relative difference of 93\% between the conditions of max (Aug) and min (Feb) MSW productions. This fluctuation of MSW production in the macro-area "Coastal Municipalities" is referable to the temporal impact of the pertinent touristic flux, as shown in Figure 4 by the qualitative correspondence of increasing and decreasing phases between the monthly evolutions of MSW production and tourist flux.

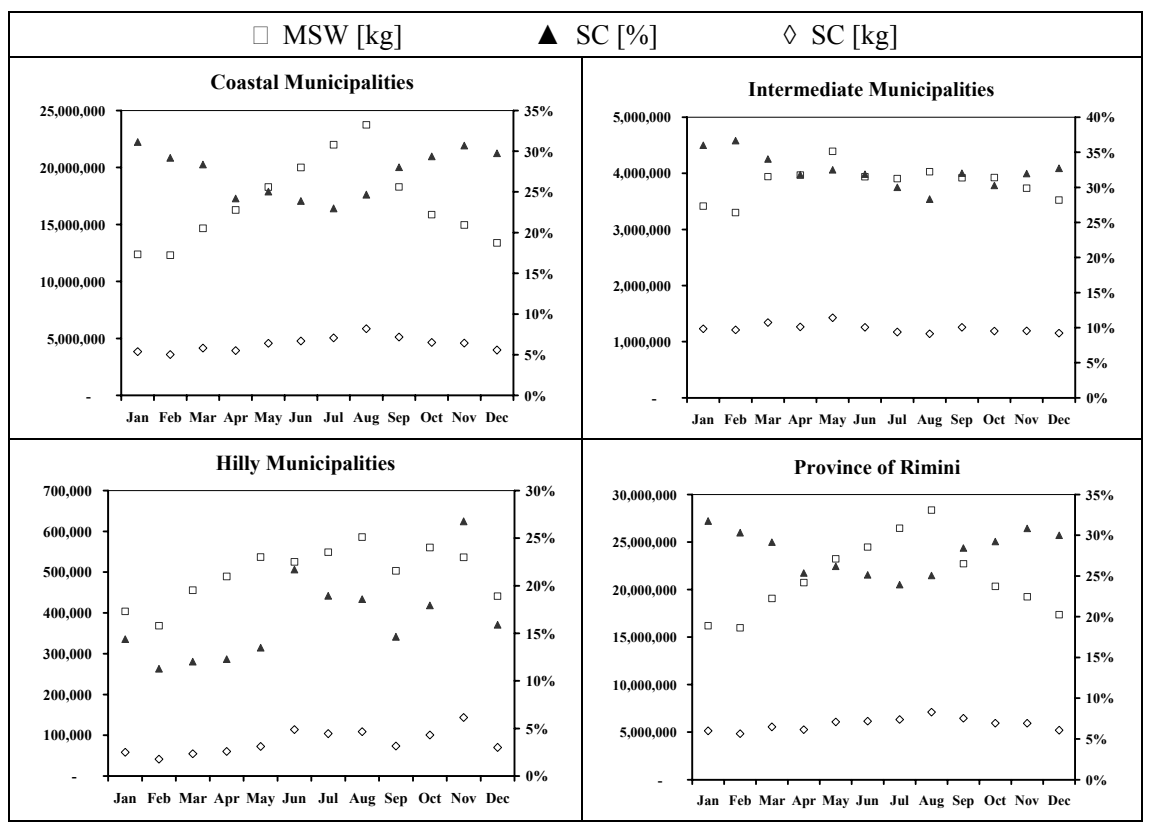

Figure 5: Monthly evolutions of MSW production and separate collection (SC, as both weight quantity and efficiency percentage) for each territorial macro-area, monitoring year 2006.

Regarding the MSW per-capita production referable on the whole to a given territorial area, the resulting values for the monitoring year 2006 are reported in Figure 6, in accordance with the alternative calculation approaches (see Section 2.2) respectively based on the sole "resident" population (diagram at left-side) and on the comprehensive "total equivalent" population (diagram at right-side). The data of Figure 6 (left-side) comparatively show a clear overestimate of the overall MSW per-capita production resulting for the touristic macro-area "Coastal Municipalities", with a relative increase of $30.3 \%$ as compared with the corresponding MSW per-capita production of the neighbouring "Intermediate Municipalities". Differently, the diagram of Figure 6 (right-side), taking into account also the "equivalent tourist" population (see Section 2.2), provide for a more realistic MSW per-capita production referable on the whole to the touristic 
macro-area "Coastal Municipalities". A relative increase only limited to $15.2 \%$ is still resulting in the comparison of the MSW per-capita production of Figure 6 (right-side) for "Coastal Municipalities" with the corresponding MSW per-capita production referred to the remaining "Hinterland" territory. Indeed, the circumstance of a higher MSW per-capita production referable on the whole to the touristic macro-area "Coastal Municipalities", as compared with the remaining provincial territory, is a reliable effect of the predominant concentration of the summer touristic fluxes in this coastal area.
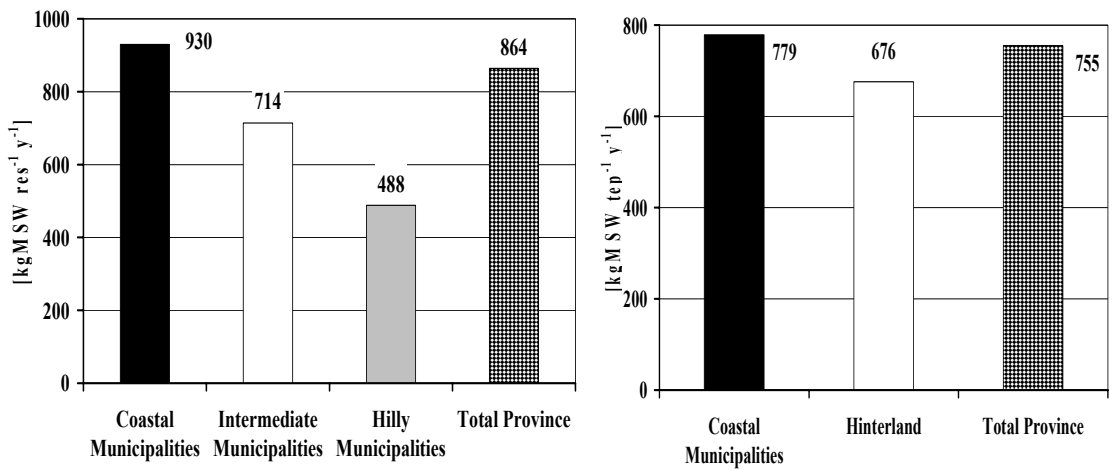

Figure 6: $\quad$ Per-capita MSW productions globally referable to each territorial area (year 2006), according to the alternative calculation approaches (see Section 2.2) of the "resident" population (leftside) and the "total yearly equivalent" population (right-side). Legend: $y=$ year; res $=$ resident; tep $=$ total equivalent population.

According to the calculation approach of Figure 2, the resulting MSW percapita productions individually attributable to the "resident" and the definable "yearly equivalent tourist" persons (see Section 2.2), for the macro-area "Coastal Municipalities" during the monitoring year 2006, are reported in Figure 7. Interestingly, Figure 7 shows a higher MSW per-capita production attributable to the "equivalent tourist" person as compared with the typical "resident" person.

Differently, the mentioned Italian study [6] on the tourism impact in the summer touristic area of a mountain valley district in the Province of Trento (North-eastern Italy: see Section 1), determined a lower MSW per-capita production for the tourist-type person as compared with the resident-type person. Besides the different calculation approaches adopted in this paper (Figure 2) and in the mentioned study [6], this discrepancy resulting with the opposite comparative condition of Figure 7 can likely be explained by the following reliable consideration: a typical tourist person spending summer holidays in a mountain locality is presumably characterised by an higher "intrinsic" environmental sensitivity and an expected, thrifty style of summer life as compared with a typical tourist person having holidays by the sea in a coastal area (especially if internationally renowned as a major summer destination). Indeed, the estimate of Figure 7 on the MSW per-capita production attributable 
to the tourist-type person, which on a daily basis corresponds to $2.6 \mathrm{~kg}$ MSW person $^{-1}$ day $^{-1}$, has a certain reliability in accordance with the following comparative evaluations: 1) this estimate (on the daily basis) is within the generic range 2-4 $\mathrm{kg} \mathrm{MSW}$ person $^{-1}$ day $^{-1}$ of tourist waste production, as derivable from the aggregation of two different literature indications $[9,10] ; 2$ ) the same estimate is close to the recent (2004) determination of $2.27 \mathrm{~kg}$ MSW person $^{-1}$ day $^{-1}$ as tourist waste production for "Numana" municipality in the "Conero Riviera" (Adriatic Sea, Central Italy, in the south of the Province of Rimini) [11], with a resulting relative difference limited to $14.5 \%$.

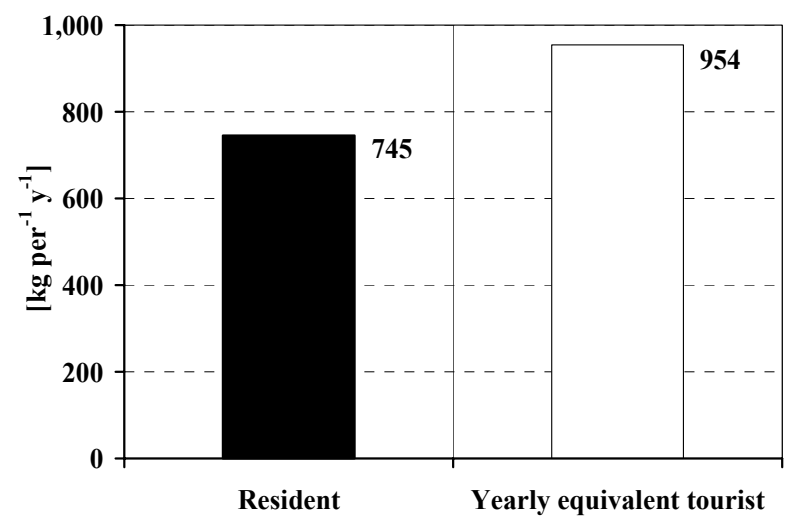

Figure 7: MSW per-capita productions (2006) individually attributable to the "resident" person and the "yearly equivalent tourist" person in the "Coastal Municipalities" area. Legend: y = year; per = person.

In accordance with the elaboration procedure summarised in Section 2.2, Table 1 shows the estimates of MSW physical composition (as component percentage by weight) resulting at the generation source in the touristic area "Coastal Municipalities", respectively for a winter month (Feb 2005), a typical summer two-monthly period (Aug-Sep 2005), and the yearly average condition. Referring to the organic fraction, the corresponding data in Table 1 show an higher incidence during the touristic summer months (Aug-Sep) as compared with a typical winter month (Feb) and also the yearly average condition; this comparative circumstance could likely be explained by the intense summer impact of hotel, restaurant and refreshment activities, with an expected, consistent generation of organic waste from cleaning and/or discarding of vegetables, fruit and foods. Similarly, the data in Table 1 show a higher incidence of the glass component during the summer months (Aug-Sep) as compared with the winter month (Feb) and the yearly situation, which is also presumably attributable to the summer tourism impact. Additionally, the data in Table 1 show a lower incidence of both the cellulosic and plastic components during the summer months; this condition could likely be explained by the expected practice, in the summer-time, of goods purchase wholesale (and not at retail) for the mentioned hotel, restaurant and refreshment activities, thus reducing/limiting the generation of small and numerous packaging wastes. 
Table 1: $\quad$ Estimates of MSW physical composition at the generation source in the macro-area "Coastal Municipalities", monitoring year 2005.

\begin{tabular}{|c|c|c|c|}
\cline { 2 - 4 } \multicolumn{1}{c|}{} & Feb 2005 & $\begin{array}{c}\text { Aug-Sept } \\
2005\end{array}$ & $\begin{array}{c}\text { Average } \\
2005\end{array}$ \\
\hline Waste component & \multicolumn{3}{|c|}{$[\%$, by weight $]$} \\
\hline Organic fraction & 24.8 & 39.4 & 28.0 \\
\hline Paper and cardboard, wood & 37.9 & 22,9 & 34,1 \\
\hline Plastics & 18.9 & 7.7 & 18.8 \\
\hline Glass & 7.4 & 11.2 & 7.9 \\
\hline Commingled metals & 2.3 & 2.5 & 3.5 \\
\hline Non considerable fractions & 8.7 & 16.3 & 7.7 \\
\hline Total & 100 & 100 & 100 \\
\hline
\end{tabular}

With regard to the separate collection (SC) of MSW in the different territorial macro-areas, the resulting monthly variations (as both weight and percentage, for the monitoring year 2006) are reported in the condensed diagrams of the previous Figure 5, in comparative representation with the monthly MSW productions. Among the municipal areas "Coastal Municipalities", "Intermediate Municipalities" and "Hilly Municipalities", the monthly variations of Figure 5 show precisely for "Coastal Municipalities" a qualitative concordance of the respective increasing and decreasing phases between MSW production and weight SC temporal evolutions. Such concordance is also confirmed by the coincidence, resulting only in the area "Coastal Municipalities" (as compared with the two remaining municipal areas), of the months with max value (precisely, August) and min value (precisely, February) for MSW production and weight SC. However, the resulting relative difference between the max and min monthly values of weight SC in "Coastal Municipalities", namely 63\%, is considerable lower than the corresponding relative max-min difference for MSW production in the same macro-area (namely 93\%, as above mentioned). Consequently, the corresponding diagram of Figure 5 shows a decreasing of SC efficiency values (as percentage) during the summer-time (especially June, July and August) in "Coastal Municipalities", which is realistically attributable to the consistent tourism impact in this area. Further, the monthly evolutions in Figure 5 of MSW production and SC efficiency (as percentage) for "Coastal Municipalities" show a "specular" accordance between the convex and concave patterns resulting respectively for MSW production and SC efficiency.

Finally, Figure 8 reports and compares the resulting estimates of per-capita weight SC yields individually attributable to the "resident" (considering both the total collection scheme or the efficiency solely from territorial road containers: see Section 2.1) and the "yearly equivalent tourist" in the macro-area "Coastal Municipalities", in accordance with the adopted estimation approach of Figure 3. Interestingly, the data in Figure 8 show clearly a decreased "inclination" towards the separate collection attributable to the "yearly equivalent tourist" person, even considering the more appropriate comparison with the same road container collection scheme for the "resident" person. This comparative circumstance 
could have the following explanations: 1) the evident, "intrinsic" difficulty for the tourist to adapt himself immediately and be integral part of the host territorial district [6]; 2) the "objective" difficulty for the touristic structures (hotels, restaurants, refreshment rooms, bathing establishments) to achieve a high efficiency of SC in the presence of an intense summer touristic flux (see Figure 4). As an indirect confirmation of a certain reliability for the adopted calculation approach of Figure 3, it should be pointed out that the estimates in Figure 8 are within the overall range of per-capita weight SC yields officially documented for the Italian Regions during the same reference year 2006 [12].

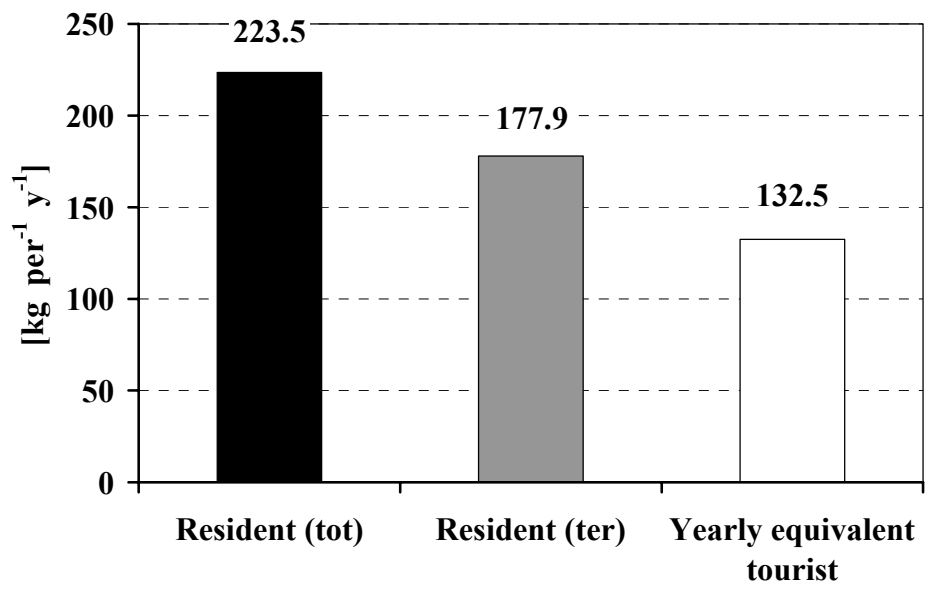

Figure 8: Per-capita weight yields of MSW separate collection (2006) attributable to the "resident" person and the "yearly equivalent tourist" person in the macro-area "Coastal Municipalities". Legend: $\mathrm{y}=$ year; per $=$ person; tot $=$ total separate collection scheme; ter $=$ territorial separate collection with road containers.

\section{Conclusions}

Referring to a representative seaside territorial district, this study has shown and quantified the summer tourist impact on MSW monthly production, composition (between winter and summer periods) and monthly separate collection yield. A further study step could specifically deal with the extension of data elaboration to the monitoring years 2007-2008. Particularly, the overall separate collection efficiency has reached $41.8 \%$ in the Province of Rimini during last year (2008).

\section{References}

[1] de Fraja Frangipane, E., Sustainability of the integrated management systems of MSW, Integrated Management of MSW, Environmental Series, Volume 26, C.I.P.A. Publisher, Milan, Italy, 2004 (in Italian).

[2] Commission of the European Communities, Communication of the Commission on the Review of the Community Strategy for Waste 
Management, COM(96) 399 final, Brussels, 30.07.1996.

[3] Directive 2008/98/EC on waste and repealing certain Directives, Official Journal of the European Union, L 312/3-30, 22.11.2008.

[4] Commission of the European Communities, Towards a thematic strategy on the prevention and recycling of waste, Communication $\operatorname{COM}(2003) 301$ final, Brussels, 27.5.2003.

[5] United Nations Commission for Sustainable Development (UN-CSD), Plan of Implementation of the Johannesburg World Summit on Sustainable Development, available at http://www.un.org/esa/sustdev/documents/, consultation January 2009.

[6] Ragazzi, M., Baratieri, M. \& Salvaterra, M., Assessment and characterisation of the tourist contribution on MSW production: a case study in the Province of Trento, RS - Rifiuti Solidi, Vol. XVIII, No. 6, pp. 373-378, 2004 (in Italian).

[7] Province of Rimini, Production, disposal and separate collection of waste in the Province of Rimini: Report 2005, Provincial Department of the Environment and the Sustainable Development Policy in cooperation with the Provincial Observatory on Waste, Rimini, Italy, July 2006, available at www.ambiente.provincia.rimini.it (in Italian).

[8] Patterson, T.M., Niccolucci, V. \& Bastianoni, S., Beyond "more is better": Ecological footprint accounting for tourism and consumption in Val di Merse, Italy, Ecological Economics, 62, pp. 747-756, 2007.

[9] United Nations Environment Programme (UNEP), A manual for water and waste management: What the tourism industry can do to improve its performance, Division of Technology, Industry and Economics Production and Consumption Branch, with the support of "GTZ, Deutsche Gesellschaft für Technische Zusammenarbeit”, Paris (France) \& Eschborn (Germany), 2003.

[10] The Heritage Environmental Rating Programme, The environmental impacts of tourism, "IAIAsa, International Association for Impact Assessment (South Africa)", accessed at www.iaia.co.za/File_Uploads/ file/TheEnvironmentalImpactsOf\%20Tourism27June2007.pps, consultation January 2009.

[11] ASTA (Actions for tourism sustainability in the Adriatic Area), A common strategy for tourism development in the coastal areas of the Adriatic Sea, edited by Michelangeli, G., Sampaolesi, S. \& Satta, A., co-financed by ERDF within the Framework of Adriatic Cross Border Interreg IIIA Programme, Marche Region, Council Department of Environment, Division of Environment and Landscape, Unit Environment Safeguard, Sustainability and Cooperation, 2006.

[12] Italian Environmental Protection Agency and Technical Services (APAT), Waste Report 2007, Department of Environmental State and Metrology, Rome, Italy, January 2008, available at www.apat.gov.it (in Italian). 